\title{
Küçük Hacimli Popüler Müzik Üretim Mekanlarının Akustik Özelliklerinin Belirlenmesi Üzerine Bir Uygulama Örneği
}

\author{
Feridun ÖZiş *
}

\section{Özet}

Mekan akustiği üzerine yapılan çalışmalar içerisinde popüler müzik eserlerinin seslendirme mekanlarına (bar, club vb.) ait araştırmalar, literatürde oldukça az yer kaplamaktadır. Ancak, dinleyici sayıları göz önüne alındığında bu mekanlar uluslararası sanat müziği mekanlarına göre çok daha kalabalık mekanlardır. Bu nedenle bar-club gibi mekanların akustik özellikleri, yapılan performansın doğru bir şekilde dinleyiciye ulaşması bakımından oldukça önemlidir.

Bu çalışma, örnek mekanlarda gerçekleştirilen ölçümlerden elde edilen veriler çerçevesinde, bu mekanların akustik özelliklerinin ortaya konması ve mekanın ölçülen akustik verileri ile seslendirme sisteminden elde edilen verilerin karşılaştırılması üzerine temellenmiştir. Bu sayede bu tip mekanlarda akustik tasarım ve ses sitemi arasındaki ilişkilerin gözlemlenmesi amaçlanmaktadır.

Anahtar Sözcükler: Akustik, Oda Akustig̈i, Müzik Üretim Mekanları, Popüler Müzik

\section{An Application for the Determination of the Acoustical Properties of Small Volume Popular Music Venues}

\section{Abstract}

Researches on the acoustical properties of popular music venues (bars, clubs etc.) are rather limited in the related literature. However, taking the number of listeners into account, these venues are more crowded than concert halls where classical music is being played. Therefore, the acoustical properties of these venues, such as bars-clubs, are quite important in respect to transmit the musical performance to the listeners properly.

This study is based on the presentations of the acoustical properties of selected venues and the comparisons of the data acquired from the sound systems with the measured acoustical data. Thus, it is aimed to analyze the relationships between the acoustical designs and sound systems in such venues.

Keywords: Acoustics, Room Acoustics, Concert Spaces, Popular Music. 


\section{Giriş}

Dünyada konser mekanları üzerine yapılan çalışmalar özellikle uluslararası sanat müziḡi seslendirilen mekanlar çerçevesinde ele alınmıştır. Bu perspektifte Beranek (2004: 19) ile başlayan akustik parametre değerlendirmeleri, günümüzde birçok konser salonunun akustik analizi/ tasarımı ile devam etmektedir. Salonların akustik performansları yaygın olarak simülasyon yazılımlarından elde edilen deḡerler ve mekan içi ölçümlemeler çerçevesinde gerçekleştirilmektedir.

Günümüzde popüler müzik mekanlarında gerçekleştirilen konser sayısının klasik müzik eserlerinin seslendirmelerinden çok daha fazla olduğu bilinen bir gerçektir. Özellikle Türkiye söz konusu olduğunda, bu farkın daha da açıldığı söylenebilir. Yapılan bir araştırmada, Danimarka'da 2004 senesinde 12.500 popüler müzik konseri gerçekleştirilirken aynı yıl 2000 klasik müzik konseri gerçekleştirildiḡi belirlenmiştir (Adelman-Larsen, 2007: 2).

Adelman-Larsen, N. W., Thompson, E. R., Gade, A. C. tarafından Danimarka'daki mekanlarda gerçekleştirilen araştırmada 'en iyi' olarak değerlendirilen mekanların 0.6-1.2sn yansışım süresine sahip oldukları belirtilmiştir (Adelman-Larsen vd., 2010: 4). Bu mekanlar 1000-6000 $\mathrm{m}^{3}$ arasında deḡişen hacimlere sahiptir. Yapılan bir başka çalışmada mekanların bas berraklık (clarity) değerlerinin yüksek olması gerekliliği ortaya konmuştur (AdelmanLarsen, Thompson, 2008: 5). Aynı çalışmada, mekanların bas yansışım süresi deḡerinin olabildiğince düşük olması gerektiği de belirtilmiştir.

\section{Ölçüm Prosedürü}

Ölçümler İzmir'de bulunan dört farklı rock barda gerçekleştirildi. Ölçüm için $288 m^{3}-400 m^{3}-486 m^{3}$ ve $800 \mathrm{~m}^{3}$ 'lük dört farklı mekan seçildi. Ölçümün hassasiyeti açısından ölçümler mekanlar boşken yapıldı. Ölçümlerde kullanılan sinyal yolunda B\&K küresel (omnidirectional) hoparlör, Dirac 6.0 ${ }^{1}, \mathrm{NTI}$ XL2 ${ }^{2}$ ve buna baḡlı Tip 1 mikrofon bulunmaktadır (Şekil 1). Kullanılan tüm ekipmanlar uluslararası akustik standartları sağlamaktadır.

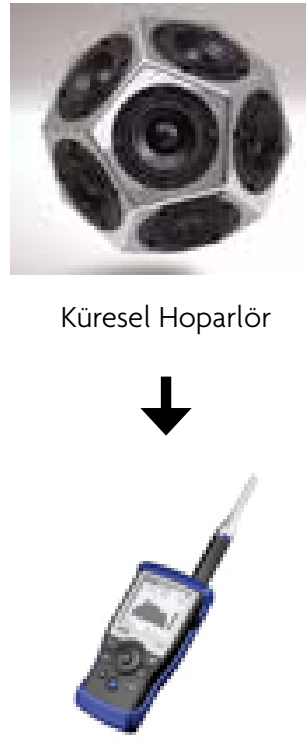

NTI Oda Analizörü

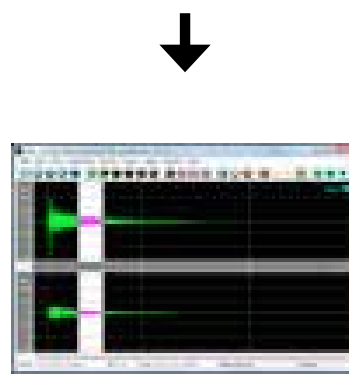

Dirac Oda Akustiği Yazılımı

Şekil 1. Ölçüm Sinyal Yolu.

Kaynak alıcı lokasyonu olarak tüm mekanlarda hoparlör kümelerine yakın ve hoparlör kümelerinden uzak iki alıcı noktası belirlendi. Kaynak noktası olarak sahnede solistin bulunduğu bölge tercih edildi. Kaynak ve alıcılar ISO $3382^{3}$ standartlarında belirtilen 1,2 m kaynak ve 1,5 m alıcı yüksekliği olarak seçildi. Her iki alıcı noktası içinde tarama (sweep) sinyal gönderilerek mekanların ani tepkileri (impulse response) elde edildi. Daha sonra, mekanda bulunan ses sisteminden sweep sinyal verilerek aynı ölçüm tekrarlandı. Her iki ölçümden elde edilen ani tepkiler Dirac programına girilerek ISO 3382 standartları kapsamında akustik veriler karşılaştırıldı (Şekil 2). 


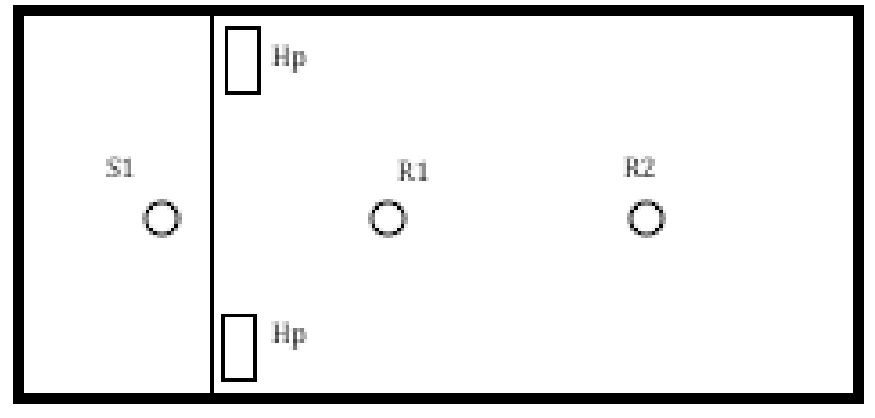

Şekil 2. Kaynak Alıcı Lokasyonları.

\section{Mekanların Ayrıntılı Analizi}

\section{No'lu Mekan}

1 no'lu mekan verileri incelendiğinde, akustik ölçüm deḡerlerinde R1 ve R2 alıcı noktaları arasında EDT parametresinin, $1 \mathrm{~K}$ ve $2 \mathrm{~K}$ oktav bantlarda $1.5 \mathrm{sn}$ seviyesinde daha yüksek olduğu gözlemlenmiştir. Mekanın arka bölümünün bu frekanslardaki EDT süresinin yüksek olması nedeniyle, bu frekanslara denk gelen enstruman tınılarının daha önde duyulmasına neden olacağı açıktır. Bu da mekandaki tonal dengeyi iki alıcı noktasında farklılaştıracaktır (Tablolar 1).

Tablolar 1. 1 No'lu Mekan Ölçüm Sonuçları

\begin{tabular}{|l|l|l|l|l|l|l|l|}
63 & 125 & 250 & 500 & 1000 & 2000 & 4000 & 8000
\end{tabular}

\begin{tabular}{c|c|c|c|c|c|c|c|c}
\hline $\begin{array}{c}\text { EDT } \\
\text { [s] }\end{array}$ & 1,69 & 0,58 & 0,52 & 0,69 & 0,81 & 0,79 & 0,71 & 0,58 \\
\hline $\begin{array}{c}\text { T30 } \\
\text { [s] }\end{array}$ & 1,85 & 0,48 & 0,66 & 0,75 & 0,83 & 0,85 & 0,77 & 0,67 \\
\hline $\begin{array}{c}\text { D50 } \\
\text { [-] }\end{array}$ & 0,52 & 0,69 & 0,77 & 0,61 & 0,61 & 0,61 & 0,66 & 0,69
\end{tabular}

R1 Alıcı Noktası Akustik Verileri

\begin{tabular}{|l|l|l|l|l|l|l|l|}
63 & 125 & 250 & 500 & 1000 & 2000 & 4000 & 8000
\end{tabular}

\begin{tabular}{c|c|c|c|c|c|c|c|c|}
\hline $\begin{array}{c}\text { EDT } \\
\text { [s] }\end{array}$ & 0,3 & 0,58 & 0,47 & 0,61 & 0,67 & 0,69 & 0,66 & 0,56 \\
\hline $\begin{array}{c}\text { T30 } \\
\text { [s] }\end{array}$ & 0,85 & 0,55 & 0,56 & 0,71 & 0,87 & 0,84 & 0,73 & 0,68 \\
\hline $\begin{array}{c}\text { D50 } \\
\text { [-] }\end{array}$ & 0,91 & 0,38 & 0,78 & 0,68 & 0,62 & 0,6 & 0,69 & 0,7 \\
\hline
\end{tabular}

R2 Alıcı Noktası Akustik Verileri

\begin{tabular}{|c|c|c|c|c|c|c|c|c|}
\hline & $\mathbf{6 3}$ & $\mathbf{1 2 5}$ & $\mathbf{2 5 0}$ & $\mathbf{5 0 0}$ & $\mathbf{1 0 0 0}$ & $\mathbf{2 0 0 0}$ & $\mathbf{4 0 0 0}$ & $\mathbf{8 0 0 0}$ \\
\hline EDT & & & & & & & & \\
[s] & 0,61 & 0,43 & 0,31 & $\mathbf{0 , 2 3}$ & 0,69 & 0,72 & 0,75 & 0,63 \\
\hline $\begin{array}{c}\text { T30 } \\
\text { [s] }\end{array}$ & 0,46 & 0,58 & 0,57 & 0,72 & 0,84 & 0,84 & 0,79 & 0,64 \\
\hline D50 & & & & & & & & \\
\hline [-] & 0,84 & 0,71 & 0,88 & 0,91 & 0,85 & 0,83 & 0,76 & 0,88 \\
\hline
\end{tabular}

R1 Alıcı Noktası Ses Sistemi Verileri

\begin{tabular}{|c|c|c|c|c|c|c|c|c|}
\hline & 63 & 125 & 250 & 500 & 1000 & 2000 & 4000 & 8000 \\
\hline $\begin{array}{c}\text { EDT } \\
\text { [s] }\end{array}$ & 0,32 & 0,34 & 0,48 & 0,52 & 0,44 & 0,54 & 0,46 & 0,34 \\
\hline T30 & & & & & & & & \\
[s] & 0,66 & 0,59 & 0,51 & 0,76 & 0,81 & 0,79 & 0,74 & 0,59 \\
\hline D50 & & & & & & & & \\
\hline [-] & 0,88 & 0,8 & 0,73 & 0,72 & 0,81 & 0,76 & 0,8 & 0,87 \\
\hline
\end{tabular}

R2 Alıcı Noktası Ses Sistemi Verileri

$63 \mathrm{~Hz}-125 \mathrm{~Hz}-250 \mathrm{KHz}$ oktav bantlardaki akustik ölçümlerde sahne önünde elde edilen EDT ortalaması 0.9 sn iken arka bölümde bu değerlerin 0.46sn seviyesine düştüğü gözlemlendi. Ön bölümden elde edilen deḡerler bu büyüklükteki bir hacim için oldukça yüksek değerlerdir. Özellikle akustik ölçümlerde $63 \mathrm{~Hz}$ oktav bant EDT değeri 1.6sn çıkmaktadır. Bu kabul edilebilir sınırların çok ötesinde bir deḡer olarak karşımıza çıkar

\section{No'lu Mekan}

Ölçülen mekanlar arasında parametre değişimlerinin en az olduğu mekandır. Akustik ölçümlerde mekan $63 \mathrm{~Hz}$ ve $125 \mathrm{~Hz}$ oktav bantta, yakın ve uzak alıcı noktaları arasında 0.45sn'lik bir değişim gözlemlenmiştir. Bu fark, mekana yerleştirilen R2 alıcısının mekanın köşe bölümüne yakın olmasından kaynaklanır. Aynı deḡer, ses sisteminden yapılan ölçümlemelerde hoparlör yayılım diyagramı sebebiyle ortadan kalkmıştır. Ölçümlerde T30 değerlerinin EDT deḡerlerine yakın çıkması mekanın RT değerlerinin düzenli olduğuna dair bir işaret olarak kabul edilebilir (Tablolar 2). 
Tablolar 2. ikinci Mekan Ölçüm Sonuçları

\begin{tabular}{|l|l|l|l|l|l|l|l|}
63 & 125 & 250 & 500 & 1000 & 2000 & 4000 & 8000 \\
\hline
\end{tabular}

\begin{tabular}{c|c|c|c|c|c|c|c|c}
\hline $\begin{array}{c}\text { EDT } \\
\text { [s] }\end{array}$ & 0,37 & 0,26 & 0,48 & 0,40 & 0,412 & 0,37 & 0,4 & 0,37 \\
\hline $\begin{array}{c}\text { T30 } \\
\text { [s] }\end{array}$ & 0,54 & 0,48 & 0,53 & 0,44 & 0,39 & 0,38 & 0,38 & 0,35 \\
\hline $\begin{array}{c}\text { D50 } \\
\text { [-] }\end{array}$ & 0,96 & 0,87 & 0,79 & 0,79 & 0,78 & 0,85 & 0,84 & 0,85 \\
\hline
\end{tabular}

R1 Alıcı Noktası Akustik Verileri

\begin{tabular}{l|l|l|l|llll}
63 & 125 & 250 & 500 & 1000 & 2000 & 4000 & 8000
\end{tabular}

\begin{tabular}{c|c|c|c|c|c|c|c|c}
\hline $\begin{array}{c}\text { EDT } \\
\text { [s] }\end{array}$ & 0,57 & 0,69 & 0,42 & 0,41 & 0,36 & 0,38 & 0,33 & 0,33 \\
\hline $\begin{array}{c}\text { T30 } \\
\text { [s] }\end{array}$ & 0,37 & 0,59 & 0,53 & 0,46 & 0,41 & 0,39 & 0,37 & 0,35 \\
\hline $\begin{array}{c}\text { D50 } \\
\text { I-] }\end{array}$ & 0,8 & 0,77 & 0,83 & 0,8 & 0,86 & 0,86 & 0,9 & 0,9 \\
\hline
\end{tabular}

R2 Alıcı Noktası Akustik Verileri

\begin{tabular}{|l|l|l|l|l|l|l|l|}
63 & 125 & 250 & 500 & 1000 & 2000 & 4000 & 8000
\end{tabular}

\begin{tabular}{c|c|c|c|c|c|c|c|c}
\hline $\begin{array}{c}\text { EDT } \\
\text { [s] }\end{array}$ & 0,40 & 0,39 & 0,39 & 0,34 & 0,38 & 0,29 & 0,33 & 0,29 \\
\hline $\begin{array}{c}\text { T30 } \\
\text { [s] }\end{array}$ & 0,59 & 0,54 & 0,58 & 0,43 & 0,37 & 0,40 & 0,38 & 0,34 \\
\hline $\begin{array}{c}\text { D50 } \\
\text { [-] }\end{array}$ & 0,9 & 0,81 & 0,87 & 0,85 & 0,86 & 0,93 & 0,91 & 0,93 \\
\hline
\end{tabular}

R1 Alıcı Noktası Ses Sistemi Verileri

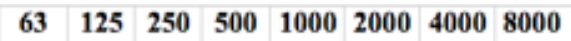

\begin{tabular}{c|c|c|c|c|c|c|c|c}
\hline $\begin{array}{c}\text { EDT } \\
\text { [s] }\end{array}$ & 0,47 & 0,22 & 0,37 & 0,33 & 0,42 & 0,35 & 0,29 & 0,24 \\
\hline $\begin{array}{c}\text { T30 } \\
\text { [s] }\end{array}$ & 0,47 & 0,51 & 0,51 & 0,42 & 0,42 & 0,38 & 0,35 & 0,33 \\
\hline $\begin{array}{c}\text { D50 } \\
\text { [-] }\end{array}$ & 0,89 & 0,96 & 0,87 & 0,89 & 0,89 & 0,89 & 0,93 & 0,95 \\
\hline
\end{tabular}

R2 Alıcı Noktası Ses Sistemi Verileri

\section{No'lu Mekan}

Bu mekanda gözlemlenen temel sorun, bitişik oktav bantlarda değişimlerin çok yüksek olmasıdır. Mekanın ön ve arka bölümlerinde gerçekleştirilen ölçümler değerlendirildiğinde arka bölümde $63 \mathrm{~Hz}-125 \mathrm{~Hz}-250 \mathrm{~Hz}$ oktav bant ortalaması 0.87 sn iken ön bölümde bu değer 0.70 sn elde edilmiştir. Bu değerler bu oktav bantlar için arka bölümdeki alıcı noktasında hacim etkisinin daha yüksek olduğunu gösterir. Mekan ortalamasına göre bu bantlar için deḡerler oldukça yüksektir (Tablolar 3).

Tablolar 3. Üçüncü Mekan Ölçüm Sonuçları \begin{tabular}{|l|l|l|l|l|l|l|l|}
63 & 125 & 250 & 500 & 1000 & 2000 & 4000 & 8000
\end{tabular}

\begin{tabular}{c|c|c|c|c|c|c|c|c}
\hline $\begin{array}{c}\text { EDT } \\
\text { [s] }\end{array}$ & 0,79 & 1,00 & 0,72 & 0,63 & 0,73 & 0,81 & 0,74 & 0,53 \\
\hline $\begin{array}{c}\text { T30 } \\
\text { [s] }\end{array}$ & 0,67 & 0,50 & 0,72 & 0,75 & 0,81 & 0,79 & 0,75 & 0,58 \\
\hline $\begin{array}{c}\text { D50 } \\
\text { [-] }\end{array}$ & 0,57 & 0,7 & 0,64 & 0,71 & 0,66 & 0,72 & 0,82 & 0,9 \\
\hline
\end{tabular}

Rl Alıcı Noktası Akustik Verileri

\begin{tabular}{l|l|l|l|l|l|l|l}
63 & 125 & 250 & 500 & 1000 & 2000 & 4000 & 8000
\end{tabular}

\begin{tabular}{c|c|c|c|c|c|c|c|c}
\hline $\begin{array}{c}\text { EDT } \\
\text { [s] }\end{array}$ & 0,62 & 0,63 & 0,85 & 0,89 & 0,74 & 0,66 & 0,70 & 0,52 \\
\hline $\begin{array}{c}\text { T30 } \\
\text { [s] }\end{array}$ & 0,42 & 0,61 & 0,71 & 0,89 & 0,84 & 0,83 & 0,75 & 0,58 \\
\hline $\begin{array}{c}\text { D50 } \\
\text { [-] }\end{array}$ & 0,79 & 0,55 & 0,63 & 0,58 & 0,76 & 0,8 & 0,71 & 0,9 \\
\hline \multicolumn{7}{r}{ R2 Alıcı Noktası Akustik Verileri } \\
\end{tabular}

\begin{tabular}{l|l|l|l|l|l|l|l}
63 & 125 & 250 & 500 & 1000 & 2000 & 4000 & 8000
\end{tabular}

\begin{tabular}{c|c|c|c|c|c|c|c|c}
\hline $\begin{array}{c}\text { EDT } \\
\text { [s] }\end{array}$ & 0,42 & 0,68 & 0,68 & 0,77 & 0,86 & 0,80 & 0,72 & 0,55 \\
\hline $\begin{array}{c}\text { T30 } \\
\text { [s] }\end{array}$ & 0,60 & 0,47 & 0,73 & 0,82 & 0,78 & 0,77 & 0,69 & 0,61 \\
\hline $\begin{array}{c}\text { D50 } \\
\text { [-] }\end{array}$ & 0,69 & 0,48 & 0,53 & 0,64 & 0,63 & 0,61 & 0,68 & 0,77 \\
\hline
\end{tabular}

R1 Alıcı Noktası Ses Sistemi Verileri

\begin{tabular}{c|c|c|c|c|c|c|c|c}
\hline & $\mathbf{6 3}$ & $\mathbf{1 2 5}$ & $\mathbf{2 5 0}$ & $\mathbf{5 0 0}$ & $\mathbf{1 0 0 0}$ & $\mathbf{2 0 0 0}$ & $\mathbf{4 0 0 0}$ & $\mathbf{8 0 0 0}$ \\
\hline $\begin{array}{c}\text { EDT } \\
\text { [s] }\end{array}$ & 0,62 & 0,73 & 0,93 & 0,96 & 0,85 & 0,82 & 0,69 & 0,6 \\
\hline $\begin{array}{c}\text { T30 } \\
\text { [s] }\end{array}$ & 0,53 & 0,64 & 0,74 & 0,83 & 0,79 & 0,81 & 0,75 & 0,64 \\
\hline $\begin{array}{c}\text { D50 } \\
\text { I-] }\end{array}$ & 0,83 & 0,47 & 0,61 & 0,57 & 0,63 & 0,63 & 0,66 & 0,71 \\
\hline
\end{tabular}

R2 Alıcı Noktası Ses Sistemi Verileri 
Ön bölümde sistemden elde edilen deḡerlerdeyse en büyük sorun 1.0sn çıkan $125 \mathrm{~Hz}$ EDT değeridir. Ancak, sönüm eğrisinin daha sonraki bölümünü açıklayan T30 parametresinde bu değer 0.5 sn seviyesine düşmüştür. Bu değerler mekanın bu bölümünde erken yansıyan seslerde bir problem olduğunu belirtir. Ayrıca sistemden elde edilen verilerde, arka alıcı noktasındaki $63 \mathrm{~Hz}-125 \mathrm{~Hz}-25 \mathrm{HHz}$ değer ortalaması 0.75 sn çıkmaktadır ki bu, bu tip mekanlar için çok yüksek bir değerdir.

\section{No'lu Mekan}

Akustik ölçümlerdeki en büyük sorun, ön bölümde $125 \mathrm{~Hz}$ EDT değerinin genel bar ortalamasının çok üzerinde $0.89 \mathrm{sn}$, ön bölümde 0.52 sn çıkan $250 \mathrm{~Hz}$ değerinin, arka bölümde 0.80 sn seviyesinde çıkmasıdır. Bu değişimlerin temel nedeni arka taraftaki alıı noktasının balkon altında kalmasındandır. Sistem performansı değerlendirildiğinde arka bölümdeki bas değerlerinin yüksek çıktığı görülmüştür. Bu, yine bu noktadaki alıcı noktasının balkon altında olmasından kaynaklanmaktadır (Tablolar 4).

Tablolar 4. Dördüncü Mekan Ölçüm Sonuçları

\begin{tabular}{|l|l|l|l|l|l|l|l|}
63 & 125 & 250 & 500 & 1000 & 2000 & 4000 & 8000
\end{tabular}

\begin{tabular}{c|c|c|c|c|c|c|c|c}
\hline $\begin{array}{c}\text { EDT } \\
\text { [s] }\end{array}$ & 0,6 & 0,89 & 0,52 & 0,68 & 0,70 & 0,68 & 0,62 & 0,44 \\
\hline $\begin{array}{c}\text { T30 } \\
\text { [s] }\end{array}$ & 0,51 & 0,66 & 0,70 & 0,76 & 0,85 & 0,83 & 0,71 & 0,56 \\
\hline $\begin{array}{c}\text { D50 } \\
\text { [-] }\end{array}$ & 0,82 & 0,79 & 0,7 & 0,62 & 0,63 & 0,66 & 0,72 & 0,79 \\
\hline
\end{tabular}

R1 Alıcı Noktası Akustik Verileri

\begin{tabular}{|c|c|c|c|c|c|c|c|c|}
\hline & 63 & 125 & 250 & 500 & 1000 & 2000 & 4000 & 8000 \\
\hline $\begin{array}{c}\text { EDT } \\
\text { [s] }\end{array}$ & 0,72 & 0,67 & 0,8 & 0,75 & 0,89 & 0,84 & 0,78 & 0,63 \\
\hline $\begin{array}{c}\text { T30 } \\
\text { [s] }\end{array}$ & 0,46 & 0,70 & 0,80 & 1,02 & 0,84 & 0,85 & 0,72 & 0,59 \\
\hline $\begin{array}{c}\text { DS0 } \\
{[-]}\end{array}$ & 0,72 & 0,43 & 0,51 & 0,55 & 0,55 & 0,56 & 0,69 & 0,72 \\
\hline
\end{tabular}

R2 Alıcı Noktası Akustik Verileri \begin{tabular}{ll|l|l|llll}
63 & 125 & 250 & 500 & 1000 & 2000 & 4000 & 8000
\end{tabular}

\begin{tabular}{c|c|c|c|c|c|c|c|c}
\hline $\begin{array}{c}\text { EDT } \\
\text { [s] }\end{array}$ & 0,28 & 0,50 & 0,68 & 0,73 & 0,68 & 0,79 & 0,56 & 0,28 \\
\hline $\begin{array}{c}\text { T30 } \\
\text { [s] }\end{array}$ & 0,38 & 0,59 & 0,72 & 0,75 & 0,74 & 0,84 & 0,71 & 0,55 \\
\hline $\begin{array}{c}\text { D50 } \\
\text { I-I }\end{array}$ & 0,91 & 0,87 & 0,58 & 0,78 & 0,8 & 0,72 & 0,88 & 0,95 \\
\hline
\end{tabular}

R1 Alıcı Noktası Ses Sistemi Verileri

\begin{tabular}{ll|l|l|l|l|l|l|}
63 & 125 & 250 & 500 & 1000 & 2000 & 4000 & 8000 \\
\hline
\end{tabular}

\begin{tabular}{l|l|l|l|l|l|l|l|l}
\hline $\begin{array}{l}\text { EDT } \\
\text { [s] }\end{array}$ & 0,66 & 0,69 & 0,79 & 0,91 & 0,74 & 0,79 & 0,65 & 0,37 \\
\hline $\begin{array}{l}\text { T30 } \\
\text { [s] }\end{array}$ & 0,61 & 0,58 & 0,71 & 0,79 & 0,86 & 0,83 & 0,75 & 0,55 \\
\hline $\begin{array}{l}\text { D50 } \\
\text { [-] }\end{array}$ & 0,78 & 0,63 & 0,62 & 0,65 & 0,74 & 0,66 & 0,69 & 0,9 \\
\hline
\end{tabular}

R2 Alıcı Noktası Ses Sistemi Verileri

Mekanda göze çarpan bir diğer sorun ise özellikle 8KHz'deki tiz frekans oktav bantın diğer bant ortalamalarına göre çok düşük çıkmasıdır. Elde edilen verilerin diğer mekanlardan elde edilen verilere göre daha yüksek çıkmasının nedeni mekanın hacimsel farkııı̆ı̆dır.

\section{Genel Deḡerlendirmeler}

Ölçülen tüm mekanlarda akustik ölçümler için kaynağa yakın alııı noktalarında $63 \mathrm{~Hz}-125 \mathrm{~Hz}$ EDT değerleri arka alıcı noktasındaki değerlere göre yüksek çıkmaktadır (Tablolar 5). Bu, mekanların bas performansının ön bölümde daha yüksek olduğunu açıklar. Bu bölümdeki sorunların ses sistemi egalizasyonu ve kaynak yönselliği sayesinde sistem tarafından düzeltilmesinin doğru bir uygulama olduğu bilinse de, ses sisteminde belirli frekansların seviyesinin azaltılması ile düşürülmesi, o frekanslara denk gelen enstruman tınılarının düşük duyulmasını sağlayacağından istenen bir durum değildir. Akustik tasarımla yapılacak bir düzeltme, sistem dengelemesinde tonmeistere daha büyük kolaylıklar sağlayacaktır.

STI (konuşma anlaşılırlığı) parametresi açısından üç mekanın ortalama değerleri 0.66-0.68 aralığında çıkarken bir mekanda bu değer 0.83 seviyesinde elde edilmiş̧ir. Bu 
mekan ayrıca EDT dağıımının en düzenli olduğu mekan olarak karşımıza çıkmaktadır. Literatür açısından 0.66 'iyi' seviyesinde değerlendirilirken, 0.83 deḡeri 'mükemmel' olarak deḡerlendirilir.

Tablo 5. Genel Ortalamaları 1 (Akustik Ölçüm)

\begin{tabular}{|l|l|c|l|}
\hline Lokasyan & $\mathbf{6 3 - 1 2 5 H z}$ & $\mathbf{2 5 0 - 5 0 0 - 1 K H z}$ & $\mathbf{2 - 4 - 8 K H z}$ \\
\hline Ön & 0.77 & 0.60 & 0.58 \\
\hline Arka & 0.60 & 0.65 & 0.59 \\
\hline
\end{tabular}

Tablo 6. Genel Ortalamaları 2 (Ses Sistemi Ölçümü)

\begin{tabular}{|l|l|l|l|}
\hline Lokasyan & $\mathbf{6 3 - 1 2 5 H z}$ & $\mathbf{2 5 0 - 5 0 0 - 1 K H z}$ & $\mathbf{2 - 4 - 8 K H z}$ \\
\hline Ön & 0.46 & 0.56 & 0.56 \\
\hline Arka & 0.50 & 0.64 & 0.46 \\
\hline
\end{tabular}

EDT ortalamaları değerlendirildiğinde ölçülen üç mekanın ortalaması 0.66-0.68sn seviyesindeyken 2 no'lu mekanda bu deḡer 0.4sn seviyesindedir. Bunun akustik olarak daha iyi sonuçlar veren bir tasarıma sahip olmasının yanında, bu mekanın en küçük hacme sahip mekan olmasıyla da ilişkili olduğu söylenebilir.

Akustik kalitenin önemli bir göstergesi olan bitişik bantların birbirine yakın EDT değerlerine sahip olması gerekliliğini 2 no'lu mekan haricinde, özellikle $63 \mathrm{~Hz}-125 \mathrm{~Hz}$ aralığında sağlanamadığı gözlemlenmiştir.

\section{Sonuç}

Popüler müzik üretim mekanlarının akustik özellikleri üzerine gerçekleştirilen çalışmalar genel hatlarıyla dinleyici memnuniyeti kapsamında değerlendirilir. Bu çerçevede literatürde büyük mekanlar üzerine yapılan araştırmalarda 800-6500 $\mathrm{m}^{3}$ 'ük hacimlerde iyi olarak deg̃erlendirilen mekanların 0,6-1.2sn aralığında T30 deḡerine sahip olduğu belirlenmiştir. Bu çalışmalarda EDT deḡeri üzerine bir araştırma gerçekleştirilmemiştir. Ayrıca, yapılan diḡer çalışmalar göstermiştir ki bu mekanlarda bas yansışım süresinin oldukça düşük olması, bas berraklık deḡerinin iyi olması açısından önemlidir.
Bu çalışma $288 m^{3}-800 m^{3}$ hacimleri aralığında bulunan daha küçük popüler müzik alanlarında gerçekleştirilmiştir. Akustik ölçümlerden elde edilen verilerde dört mekan için EDT ortalaması 0.63 sn seviyesindedir. Ses sisteminden elde edilen veriler ise 0.53 sn ortalamasındadır (Tablolar 6). Bu ses sistemi sinyal yolunda bulunan ekipmanların frekans karakteristikleri ve hoparlör yayılım diyagramlarının, parametreleri ciddi bir miktarda değiştirdiğini gösterir. Özellikle bas frekanslarda bu değişimin oldukça fazla olduğu gözlemlenmiştir. Ancak, akustik problemlerin ses sistemi tarafından azaltılması doğru bir uygulama olsa da, bunun mümkün olduğunca minimum ölçüde gerçekleştirilmesi önemlidir. Bu sayede tonmeister, problem gidermek yerine tonal sorunlarla daha rahat bir biçimde ilgilenebilir.

Verilerin analizi sonucunda, mekanların ön ve arka bölümündeki parametre deḡişimleri genel ortalamalarda görülmese de, özellikle akustik ölçümlerde ön ve arka bölüm parametreleri belirli oranlarda deḡişiklik göstermiştir.

Literatürdeki diğer çalışmalarla karşılaştırıldığında 2 no'lu mekan dışındaki tüm mekanlarda EDT parametresinin yüksek oldug̃u söylenebilir. Hacim olarak küçük olan mekan çıkartıldığında ölçülen mekanların $63-125 \mathrm{~Hz}$ EDT ortalamaları akustik ölçümde 0.92 seviyesindedir. Bu deḡer 800-6500 m³'lük hacimlerle karşılaştırıldığında oldukça yüksektir. Ayrıca, yapılan çalışmalardan çıkan en önemli sonuç olan bas yansışım süresi deḡerinin düşük olması özelliği bu mekanlarda sağlanamamaktadır. 


\section{Notlar}

1 Dirac Oda Akustiği Yazılımı, Groenling 43-45, Boxmeer, The Netherland.

2 NTI XL2 Akustik Analizör, NTI Audio

3 ISO 3382, Measurement of the reverberation time of rooms with reference to other acoustical parameters, Second edition, 1997, Switzerland.

\section{Kaynakça}

Beranek, Leo (2004). Concert Halls and Opera Houses - Music Acoustics and Architecture. New York: Springer-Verlag.

J. Adelman-Larsen, N.W., et al. (2007). "Acoustics in Rock and Pop Music Halls", Convention Paper. Audio Engineering Society: 122nd Convention, Vienna.

Adelman-Larsen, N.W., Thompson, E. R., Gade, A. C. (2010). "Suitable Reverberation Times for Halls for Rock and Pop Music". Journal Acoustic Society America 127 (1): 247-255.

Adelman-Larsen, N. W., Thompson, E. R. (2008). "The Importance of Bass Clarity in Pop and Rock Venues". Journal Acoustic Society America 123 (5): 1-6. 